Chimia 45 (1991) 109-113

(C) Schweiz. Chemiker-Verband: ISSN $0009-4293$

\title{
LC for Sample Preparation in Coupled LC-GC: A Review
}

\author{
Konrad Grob*
}

Abstract. In chromatographic trace analysis, most of the working time is spent in preparing the sample; the methods involved are largely inefficient, often rather primitive. (HP)LC could replace a good part of this pre-separation, clean-up or sample enrichment work. It offers high separation efficiency, a wide range of selectivities, and accurate control through on-line detection. LC work is reviewed that is related to on-line LC-GC, showing the wealth of possibilities available for $\mathrm{LC}$ sample preparation. The present problems are discussed, hoping to motivate colleagues getting involved in the development of advanced sample preparation techniques.

\section{More Efficient Sample-Preparation Methods}

Did the development of chromatography come to an end? Columns and instrumentation remained largely the same for several years, and if the efforts to think about better alternatives or the investment into new methods are taken as an indication of innovation, there is litt]e going on, indeed. Of course, new techniques such as super critical fluid chromatography (SFC) and capillary electrophoresis fill journals and symposia. However, for the large part of routine analyses carried out in industrial and public laboratories, these are rather exotic techniques and/or no alternatives to existing methods. Liquid and gas chromatography are the predominating chromatographic techniques and will remain so for some time. Furthermore, are the pressing problems really concerned with the final step of the chromatographic analysis?

There are many factors stopping further development of chromatography: resignation in front of frustrating problems and unreliable results; much of the development disappeared behind the thick walls of firms making their money with chromatography; decreasing knowledge by average chromatographers; finally, the overwhelming amount of literature has become indigestible for most of the newcomers.

On the other hand, analytical problems are ever more demanding: required detection limits decrease, the number of samples to be analyzed increases, the number of people available for carrying out the sample preparation work diminishes, and, of course, costs should decrease. I's there any potential left for improving chromatography? Yes, there

*Correspondence: Dr. K. Grob

Kantonales Labor

P.O. Box, CH-8030 Zürich surely is, although not primarily at the final analysis step: it is unlikely that there will ever be a technique allowing, e.g., direct introduction of a strawberry or urine into GC. We must accept that often intensive 'pre-treatment' or 'clean-up' is required prior to $\mathrm{GC}$ analysis.

\section{Sample Preparation Keeps Low Profile}

Always a large proportion of the samples required preparation prior to the final chromatographic analysis. However, it appears that sample preparation was never taken as serious as the final analysis step. A look at the methods involved creates the impression that these steps were considered provisional, a kind of makeshift, as if expecting that a future method could do without such preseparation. Few analysts appear to be proud of their sample-preparation methods, as seen by the fact that sample preparation is hardly ever part of the title of a paper. The common titles are like 'Determination of Compound $X Y$ by GC-FTIR-MS', not mentioning that the sample had to be pre-treated maybe in five steps taking a full day of manual work! Although it constitutes the major part of the analysis, sample preparation keeps low profile, presumably because methods are considered primitive - and most probably they really are.

There is a peculiar division between the highly sophisticated methods for the final analysis step and the primitive methods used for the earlier stages. It is strange to see that $10 \%$ extra separation efficiency is considered relevant for the final analysis, but nobody seems to ask about separation efficiencies of the pre-separation steps. Nobody measures numbers of theoretical plates obtained by 'filtration' through sample preparation tubes. Why don't we more intensively apply the efficient methods used for the final analysis also for sample preparation? High separation efficiency and well tunable selectivity could only be of advantage.

(High performance) liquid chromatography (LC) not only is a powerful analyti- cal method, it also allows most effective sample preparation, e.g., for GC. Effectiveness includes high separation efficiency, high speed, and, if coupled on-line, a high degree of automation, eliminating all or at least a large proportion of the sample preparation work. It might turn out to be the future technique for sample preparation in trace analysis as well as for the analyzers designed for complex analyses.

\section{Why On-Line Coupled LC-GC?}

Capillary GC has a number of important advantages: it offers extremely high separation efficiency, is a rapid separation process and can be used with a wide range of excellent detectors - e.g., coupling to MS is simple and provides high sensitivity as well as selectivity. However, GC also has an important drawback: it usually requires intensive sample clean-up, primarily because of involatile and highly polar by-products. Such 'dirt' cannot be removed from the GC system (except by replacing parts); it accumulates and finally ruins the GC system. LC is less sensitive to by-products, because their removal is easier.

As a result, $\mathrm{GC}$ is often hampered by the required intensive sample preparation, which is not only time-consuming, but also a source of error and uncertainty - whenever a result does not correspond to expectations, the analysis must be repeated.

LC was used for preparing samples for $\mathrm{GC}$ for a long time. However, the two techniques were used off-line. Off-line LC-GC can hardly be automated and essentially remains a manual method. However, the major problem of off-line LC-GC is the poor efficiency of the transfer to GC: of a l-ml LC fraction, $1-2 \mu \mathrm{I}$ are injected into $\mathrm{GC}$, and $99.8 \%$ of the sample is wasted; after reconcentration, still hardly more than a few percent of the LC fraction is transferred to GC. Usually, it is impossible to inject 100 times more sample material into LC to compensate for this loss - often the LC column is loaded up to the maximum of its capacity, even when transferring the whole fraction by on-line LC-GC. Furthermore, loading large amounts of 'dirt' onto LC columns should be avoided. Thus, the use of off-line LC-GC (or on-line transfer of $|-2-\mu|$ volumes) is restricted to relatively concentrated samples.

\section{Transfer of LC Fractions to GC}

The most important obstacle against online coupling LC to GC was the large volume of eluent to be transferred to GC - this is probably the reason why on-line coupled LC-GC did not emerge previously. For organic eluents, two transfer techniques are routinely used: concurrent eluent evaporation with the loop-type interface, and the retention gap techniques with the on-column interface (including partially concurrent eluent evaporation) [1]. Partially concurrent 
evaporation allows easy transfer of fractions of up to some $800-\mu \mathrm{l}$ volume; with concurrent evaporation, easy transfer is restricted at a few milliliters. Cortes et al. [2] used similar evaporation techniques, but another, never really disclosed interface. Heo and Suh [3] proposed the use of a vial-shaped on-line concentrator instead of the uncoated precolumn.

\section{Problems with Water-Containing LC Elu- ents}

Several attempts were made for transferring $\mathrm{H}_{2} \mathrm{O}$-containing $\mathrm{LC}$ eluents to $\mathrm{GC}$ However, when applying the currently used transfer techniques, there are problems concerning wettability of the GC pre-columns and degradation of the pre-column deactivation [4][5]. Nevertheless Duquet et al. [6] Cortes et al. 17], and Goosens et al. [8] reported some promising results. We trans ferred up to $250 \mu$ l of $\mathrm{MeOH} / \mathrm{H}_{2} \mathrm{O}$ 60:40 by concurrent evaporation [9]. Experiments on concurrent evaporation with co-solvent trapping allowed transfer of up to $\mathrm{I} \mathrm{ml}$ of $\mathrm{H}_{2} \mathrm{O}$ containing 22\% 2-butoxyethanol as cosolvent [10] [11]. Despite these encouraging results, there are doubts that such techniques are as reliable as those with eluents free of $\mathrm{H}_{2} \mathrm{O}$.

$B o o$ and Krohn [12] exchanged the aqueous mobile phase by an organic desorption medium, evaporating the residual $\mathrm{H}_{2} \mathrm{O}$ containing eluent from the LC column (displacement of the eluent by a gas removes less than half of the liquid). Van Zoonen et al. [13] and Goosens et al. [14] described experiments involving on-line extraction from an aqueous into an organic solvent by a sandwich-type phase separator, also aiming for avoiding the transfer of $\mathrm{H}_{2} \mathrm{O}$ into $\mathrm{GC}$.

There are probably as many opinions on future trends in coupling reversed phase LC to $\mathrm{GC}$ as there are people working in the field. We believe that PTV vapor overflow, described as a concept [15], will be the most robust technique, although not producing as accurate results as the on-column methods.

This survey on presently available transfer techniques shows that the choice of LC eluents is restricted. Volatile organic solvents of normal phase LC are considered easy. The most commonly used eluents are pentane, hexane, $\mathrm{Et}_{2} \mathrm{O}, t$-BuOMe, AcOEt, and cyclohexane. $\mathrm{H}_{2} \mathrm{O}$-containing eluents, i.e. most of reversed phase $\mathrm{LC}$, cannot be recommended to newcomers and might always be more difficult. Of course, salts and other involatile additives cannot be transferred to $\mathrm{GC}$.

Limited Usefulness of Reversed-Phase $L C$ in $L C-G C$

The importance of the still existing problems with reversed-phase LC-GC is usually overemphasized. In analytical LC, reversed-phase LC strongly predominates normal phase LC. However, LC for LC-GC is different, and among the many LC-GC methods developed in our laboratory, there was just one that required reversed-phase conditions, and only in one further case reversed phase LC would have been an alternative. In all other cases, reversed-phase LC was not applicable, either because of insufficient solubility of the sample matrix in the mobile phase (mostly large amounts of fat were involved), instability of derivatives prepared for GC, but also because exchange of an apolar solvent from extraction by a reversed-phase solvent would have complicated the procedure or caused loss of volatile components.

LC-GC essentially is a GC method, and is amenable only to components well passing through a GC column. Therefore, LC$\mathrm{GC}$ does not deal with highly polar solutes, nor with strong acids or bases, where reversed-phase LC has its most important advantages. There are, however, samples that are most conveniently enriched by reversed-phase $L C$, e.g. pesticide residues from $\mathrm{H}_{2} \mathrm{O}$. In this case, transfer in a reversedphase eluent is almost mandatory.

\section{Normal Phase LC for LC-GC}

Nearly all routine LC-GC applied up to today involved normal phase LC on silica gels. The mobile phases were composed of pentane or hexane, maybe with a small admixture of a more polar solvent, such as an ether, $\mathrm{CH}_{2} \mathrm{Cl}_{2}, \mathrm{MeCN}$, or i-PrOH. Typical applications included the determination of aroma components in food extracts, of pesticides or PCBs in various matrices, the analysis of minor components in edible fats and oils, pharmaceuticals in biological fluids, aromatics in fuels (summarized in [1]), the determination of polycyclic aromatics in fat/oil [16], or the analysis of mineral oil products from packaging materials, release agents, glues, or lubricant greases in foods [17] [18].

\section{Capacity of Silica Gel}

For many applications in food analysis, LC pre-separation includes the removal of large amounts of triglycerides. Whether analyzing fats, oils, or extracts from foodstuffs, such as from milk products, fish, meat, or nuts, fat is usually the strongly predominating part of the sample matrix. Achievable detection limits depend on the amount of triglycerides that can be introduced into LC without excessive column overloading. In these respects, LC for LC-GC resembles preparative $\mathrm{LC}$.

Of course, large LC columns provide increased capacity, but since the volumes of the fractions to be transferred to GC grow proportionally, we are interested in the smallest column serving the purpose and in an optimal use of the available capacity. With an alkane as mobile phase, $10 \mathrm{~cm} \times 2$ $\mathrm{mm}$ i.d. column retained up to some $25 \mathrm{mg}$ triglycerides; for $10 \mathrm{~cm} \times 5 \mathrm{~mm}$ i.d. columns breakthrough occurred at around $150 \mathrm{mg}$. The addition of more polar solvents to the mobile phase substantially reduced these capacities [19]. Capacities of derivatized silica gels are massively lower.

\section{Stability of LC Retention Times}

One of the problems in coupled LC-GC, particularly with silica gel columns, concerns the stability of the LC retention times. The high separation efficiency provided by LC can only be exploited, when fractions are cut sharply. The determination of the fraction window is greatly facilitated by the UV detector usually installed in-line between LC and GC. However, in real samples the components of interest are seldom detected (this is why GC is used), and these transfers are bound to occur blindly.

Automated transfer mostly involves absolute LC retention times for the fraction windows. This, however, presupposes stable retention times, even when loading the LC column with large amounts of 'dirt' (including varying concentrations of moisture). Usually, it is impossible to keep LC peaks at the retention times observed in the first chromatogram. It is easier to let retention times drift away and to stabilize them at some steady state conditions: LC is carried out in a well reproduced cycle involving injection, chromatography up to the transfer of the fraction(s) of interest, backflush with a wash solvent, and reconditioning with the mobile phase. Work in such cycles presupposes optimization of conditions with the real samples, and is greatly facilitated by completely automated systems.

There is an alternative to transfer at absolute retention times: if a well-detectable compound is co-eluted with the compound(s) of interest, peak detection signals from the LC detector may be used for initiating and stopping transfer to GC. If another welldetected compound is eluted prior to the fraction of interest, such signals can be used with a delay corresponding to the difference in retention times. By this way, sharp cuts of LC fractions do no longer presuppose reproducible absolute LC retention times.

Although there are possibilities of dealing with drifted or drifting LC retention times, there remains the interest in improving retention-time stability - and there certainly is some room for improvement. The choice of the wash solvent is critical: it should efficiently clean the LC column, but also be easily removed during the following reconditioning. Maybe, silica gels could be treated to permanently remove the most adsorbing sites, somewhat analogous to buffering acid/base behavior of silica gels according to Schwarzenbach [20].

\section{Mobile Phases}

In addition to the common criteria for selecting the LCeluent, there are some special aspects concerning coupling LC to GC. GC analysis is often added to LC, because LC alone does not offer sensitive detection for the components of interest (e.g. due to lack of a suitable chromophor). This is the reason 


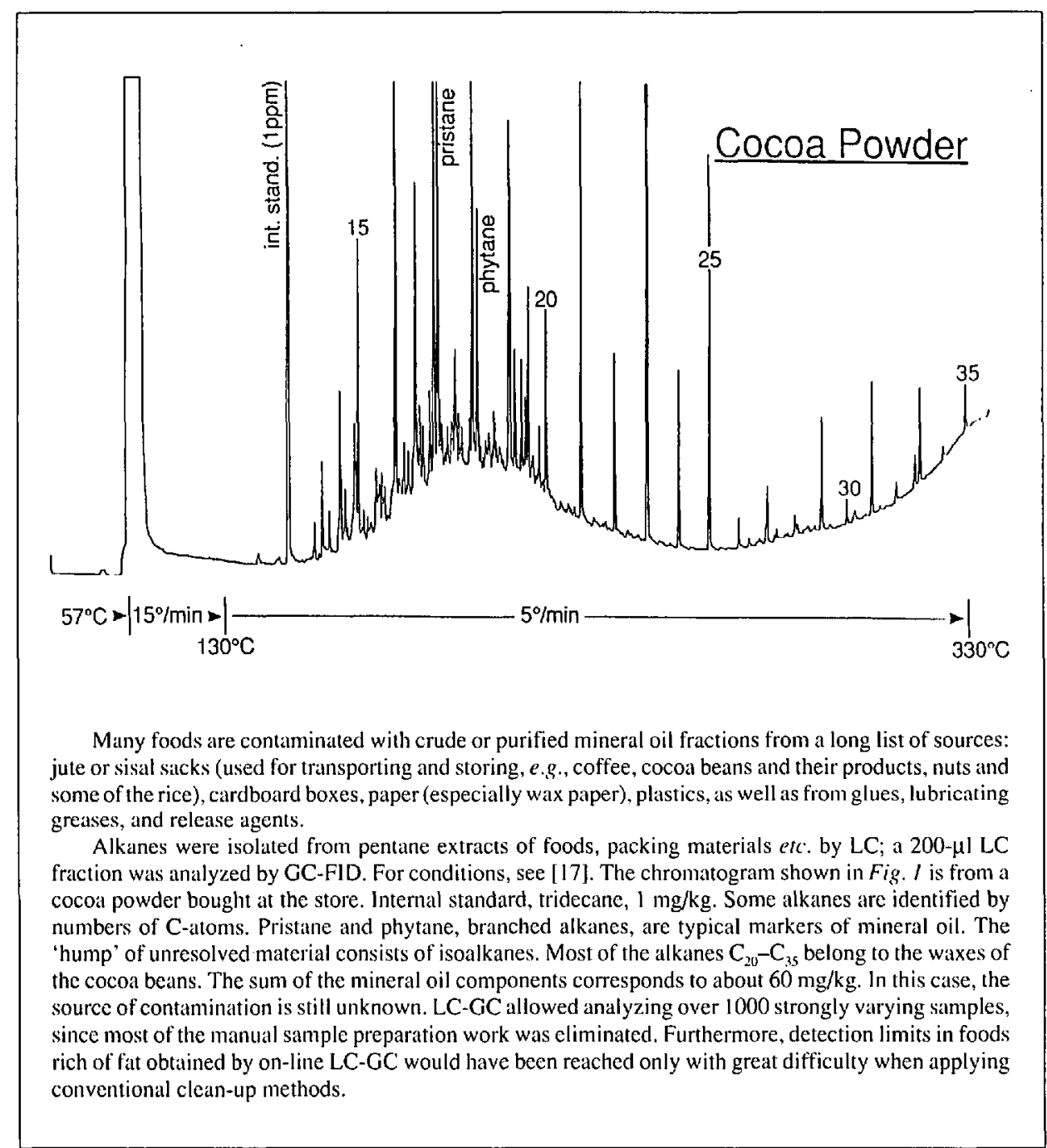

Fig. I. Analysis of mineral oil products in foodstuffs

why UV detection in LC-GC normally requires low wave lengths, which in turn restricts the selection of solvents available as mobile-phase components to those with a low UV cut-off.

An additional argument may concern suitability for transfer to GC: often concurrent evaporation is used for analyses starting at fairly low GC temperatures. This requires a volatile eluent in order to allow transfer at low oven temperature. In fact, most of these applications involve pentane as a basis of the mobile phase. However, if all components are eluted at column temperatures above $180-200^{\circ}$, or if retention gap techniques are applied, hexane may be preferable, because reduced cooling of the GC oven shortens analysis times.

The frequent use of pentane in $\mathrm{LC}$ mobile phases compels us to take measures against bubble formation: syringe pumps and/or pressurized solvent reservoirs are used, and the waste lines are equipped with flow restrictions.

The choice of more polar additives to the mobile phase is small. $\mathrm{Et}_{2} \mathrm{O}$ was widely used, also because all mixtures with pentane have virtually the same (low) boiling point as pentane and $\mathrm{Et}_{2} \mathrm{O}$ alone, facilitating the adjustment of the transfer temperature. However, at the large amounts of eluent transferred to $\mathrm{GC}$, peroxides at concentrations as
Nevertheless, for more polar components the use of $\mathrm{CN}$ columns with mobile phases of low polarity is an attractive alternative, also because lower concentrations of polar solvents are required in the eluent. Derivatized silica gels of higher polarity would be desirable to be widely applicable.

Davies et al. [23] used an amino column with pentane as mobile phase for the analysis of aromatics in diesel oil and diesel exhaust particulates. The same type of column was used for analyzing the polycyclic aromatic hydrocarbons in a higher-boiling gasoil-type mineral oil fraction used as a batching oil on jute sacks [24]. In both cases, the excellent group-type selectivity, preseparating the polyaromatics according to ring size and ring system (Wise et al. [25]) was the reason for selecting the amino phase.

Cortes et al. [2] isolated PCBs from coal tar by an ODS column in the reversed phase mode, using MeCN as mobile phase. $B a r$ carolo [24] used an ODS column with isooctane as the eluent for separating chlorinated pesticides from butter fat. With this mobile phase, all the pesticides of interest were eluted prior to the triglycerides. The triglycerides were removed from the column using hexane, as this solvent was a much stronger eluent for the fat than the branched alkane.

\section{Group-Selective Pre-separation}

LC applied for LC-GC often becomes the art of not separating the components of interest, such that a group of substances $(c . g$. of pesticides or mycotoxins) can be transferred to GC as a narrow fraction. Minimal separation in $L C$ is required for two reasons: the broader the transferred fraction, the more sample by-products are co-transferred, and the less effective was the clean-up; the high separation efficiency of LC is of little use if LC fractions consisting of many peaks are transferred to GC. Secondly, large fraction volumes cause problems for the transfer. Well-designed derivatization may solve the problem. If a group of components with identical fundamental structure but with different functional groups should be analyzed, e.g. heroin, morphine, and codeine in plasma or urine, derivatization (acylation) may render these components similar in polarity, such that they are more or less coeluted from the LC column. However, if substances differ by positional isomerism or by the number of substituents, as do the chlorinated biphenyls (PCBs), only the LC column and the eluent can be optimized. They should bring the different components closer together by an interaction concentrating on the common part of the molecules, the aromatic ring system in the case of the PCBs. If a mixture is composed of molecules with strongly differing structures, such as the chlorinated pesticides, the case is even more difficult. Since the transfer of several LC fractions is no problem, it may be preferable to transfer such mixtures in several portions for fully automated LC-GC. 


\section{Sample Enrichment in the Reversed- Phase Mode}

The fully automated water analyzer for organic trace components is the dream of many. It could be installed in the water supply line to water works and permanently analyze the incoming water for critical organic compounds. Presumably it would include sample enrichment by LC, preseparation by LC, and analysis of the fraction of interest by GC. Werkhoven-Goewie et al. [27] used very small $(2 \mathrm{~mm} \times 4.6 \mathrm{~mm}$ i.d.) $L$ C columns packed with $\mathrm{PRP}_{1}$, a styrene-divinyl-benzene copolymer, for extracting up to $I I$ of water. The final analysis was carried out by LC. Noroozian et al. [28] extracted chlorinated pesticides from $1 \mathrm{ml}$ of water using a 4 $\mathrm{mm} \times 1.1 \mathrm{~mm}$ i.d. micro-column built into a rotating switching valve. This column was packed with a $C 8$ bonded silica gel of $40-\mu \mathrm{l}$ particle size (to prevent rapid blockage by particles). After the extraction of the water the micro-column was dried in a stream of $\mathrm{N}_{2}$, and the solutes of interest were transferred on-line to GC by hexane. Vreuls et al. [29] proposed desorption from such LC enrichment columns by AcOEt, since this solvent dissolves water at a lower concentration than evaporates azeotropically in the GC pre-column.

It is not obvious that very small sample enrichment LC columns are the best choice. A $10 \mathrm{~cm} \times 2 \mathrm{~mm}$ i.d. ODS column was used for the extraction of atrazine from $10 \mathrm{ml}$ of water [9]. The extract just formed a short initial band in this column, i.e. the capacity was by far not exploited. This offered two advantages: first, the sample could be preseparated on the same column. Secondly, the higher retention power allowed the use of an eluent containing less water, facilitating the transfer to GC.

\section{Size-Exclusion \\ Chromatography (SEC)}

GC is sensitive to high-boiling or involatile sample by-products, because such material accumulates and builds up retention power retaining the solutes of interest. 'Dirty' GC column inlets cause peaks to be distorted or even let them disappear; aggressive sample by-products tend to degrade labile components. These problems are particularly severe when applying on-column injection or on-column LC-GC transfer (as are the currently used LC-GC transfer techniques). On the other hand, on-column transfer provides outstanding quantitative results [30].

SEC or gel-permeation chromatography (GPC) responds to this problem: it enables to remove those high-molecular-weight components, e.g. lipids and waxes, that cause us trouble in GC. Cleaning of such columns is simple and rapid. Specht and Tillkes [31] developed GPC to a standard method for clean-up of pesticide samples. Similar techniques were recently reported by

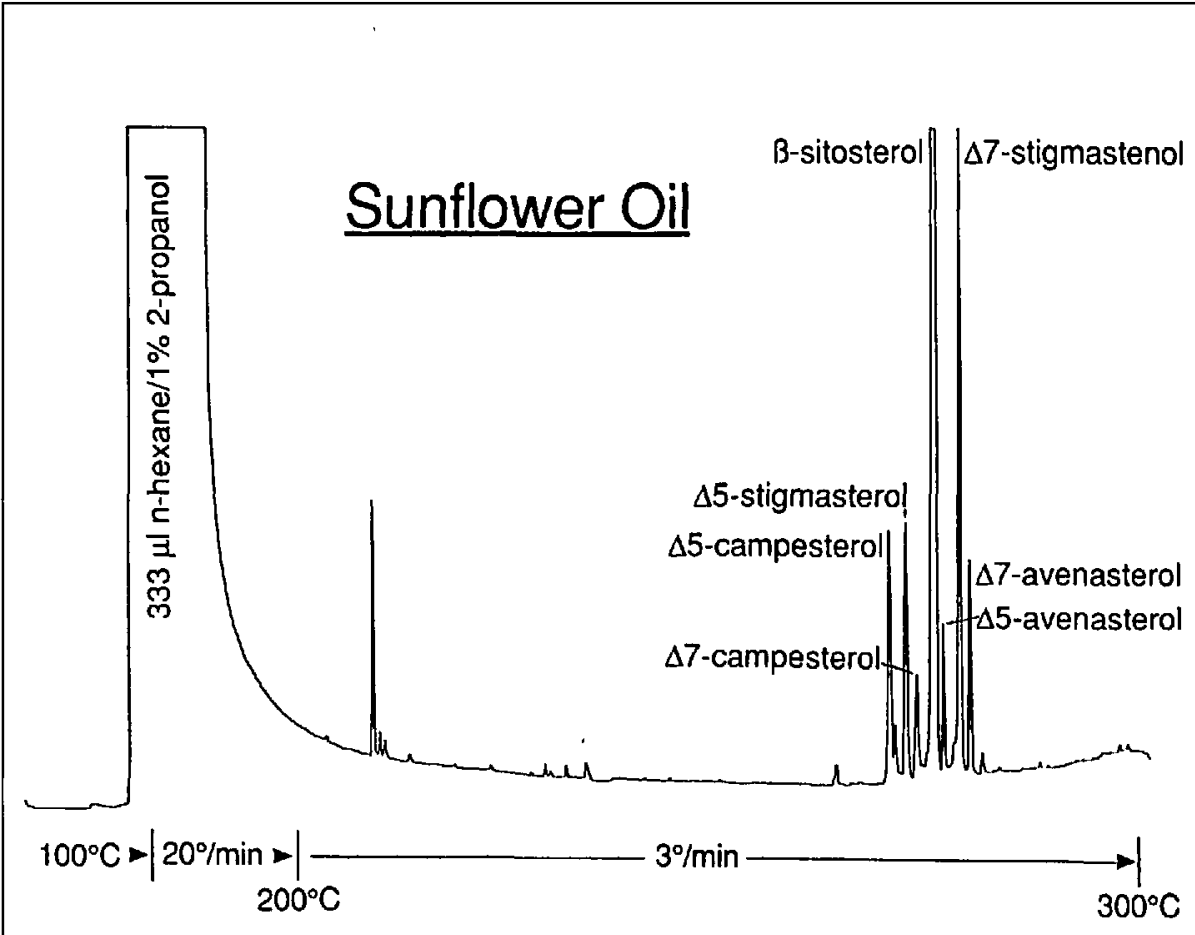

The 'sterol fraction' of the unsaponifiable of edible oils and fats is widely analyzed for confirming the identity of an oil (e.g. to rule out admixture of cheap oils to more valuable oils, such as rape seed oil to olive oil), or for checking its quality. The conventional method involves saponification of the oil, a difficult (and unreliable) extraction of the unsaponifiable from the resulting soap solution, neutralization of the extract, pre-separation by preparative TLC, silylation, and GC. Some two sample can be analyzed per person and working day.

A method allowing the analysis of minor components in their original state was described previously [43][44]. However, since most of the European laws rely on determining the sum of the free and the esterified sterols, recently a compatible LC-GC method was developed. It involves transesterification ( 15 min at ambient temperature) and automated LC-GC analysis, LC removing the methyl esters and other interfering components. Some 30 samples can be analyzed per daly and person (not considering transport of the many bottles of oil into and out of the lab and the paper work!). Results are reproducible to within some $2 \%$ (for the whole analysis), which is far better than seen previously (the extraction from the soap solution is circumvented and LC-GC transfer produces more accurate results than syringe injection into GC).

The GC chromatogram in Fig. 2 shows the LC fraction of the $\Delta 5$ and $\Delta 7$ sterols of an unrefined sunflower oil. To obtain absolute concentrations, cholesterol would be added as internal standard (eluted before $\Delta 5$ campesterol).

Fig. 2. Analysis of minor components in edible oils and fars

Chamberlain [32] and Tuinstra et al. [33]. Improved column packing materials are developed [34].

No routine on-line SEC-GC method has been described in literature, but several laboratories work on it. The columns commonly used for preparative sample preparation are far too large for direct coupling to $\mathrm{GC}$, nor is their large capacity of any use when complete fractions are transferred. Recently, Tuinstra et al. [35] described the preparation of 2-mm i.d. columns packed with Bio Beads SX-3. Fraction volumes of such columns are in the range of $200-600 \mu \mathrm{l}$ (depending on whether a single compound or a broad range, e.g., of pesticides should be transferred). This is well in the range of fraction sizes that are easily transferred to GC. Sample volumes of $c a .50 \mu \mathrm{l}$ can be injected into these columns without significant loss in separation efficiency, and the column capacity for removing large amounts of by-products seems ample.

2-3-mm i.d.columns packed with smaller particles of the same type of material are commercially available. Their separation efficiency is considerably higher, and since the polymer is of a stable volume, they allow easy exchange of the mobile phase. Most important, however, is that they are far less sensitive to pressure changes than soft polymers, such as the Bio Beads. Unfortunately, their price is still high.

The preparation of capillary SEC columns was described by Takeuchi et al. [36] and by Ghijs et al. [37]. The latter authors showed some excellent separations of compounds in the range of the molecular sizes of interest for SEC-GC applications, using columns of up to $2 \mathrm{~m}$ in length. They also described some preliminary results on SECGC of PCBs in triglycerides [38]. However, the amounts of triglycerides injected were far too small for practical use, probably because of technical problems.

Our interest in SEC primarily concerns removal of large amounts of fat, whether analyzing pesticide or veterinary drug resi- 
dues in meat. SEC might become a simple standard step in many sample preparation methods, because the same system (including the same eluent and the same fraction windows) can be used for virtually all kinds of components analyzed by GC. It also allows isolating groups of strongly differing components which partially overlap with, e.g., triglycerides and waxes when chromatographed by polarity (a common problem in food analysis).

SEC usually results in a chromatogram with a very large peak, e.g., of triglycerides eluted first, followed by the components of interest. This requires to solve a technical problem: Practically all LC peaks tail, at least when looking at a very tall peak such as the triglyceride peak in such applications. This tail reaches far into the fraction transferred to GC and may severely disturb GC.

\section{Other LC Techniques for LC-GC}

The broad range of LC separation techniques available today probably offers many more possibilities which could be exploited for LC-GC. For instance, no attempts have been made to use ion-exchange chromatography for sample clean-up coupled to GC. Coupled column LC will certainly be of interest, but little has been published on this aspect. LC-LC with backflush allows strong sample enrichment, as shown by Ramsteiner [39] in a brilliant method for the LC-GC analysis of folpet in hops.

Sample enrichment on an LC column by on-line dilution with a weak eluent was shown by Fowlis [40]. Dilution with a weak eluent (an alkane or $\mathrm{H}_{2} \mathrm{O}$ ) often causes the migration speed of solutes to be reduced more strongly than the sample volume is increased at the same time.

\section{On-Line Derivatization}

GC often requires derivatization of the components, for reducing the adsorptivity of functional groups, for bringing a group of components together into the same LC fraction, or for enhancing detector sensitivity (e.g. allowing use of ECD). For practically all LC-GC applications carried out until now, derivatization occurred prior to LC. In many cases, this derivatization also facilitated LC. In other cases, however, on-line derivatization between $\mathrm{LC}$ and $\mathrm{GC}$ would be desirable.

Raglione and Hartwick [41] described the isolation of triglycerides by LC followed by transesterification before the GC analysis. The system worked on-line, but the reaction time of $2 \mathrm{~h}$ clearly exceeded that envisaged for a routine method. On-line derivatization can be carried out within the LC system (i.e. by techniques similar to those used for post LC column derivatization) or within the GC system, as successfully used for derivatizing pentachlorophenol [42].

\section{Discussion}

Coupled LC-GC proved to be a powerful method for a broad range of applications primarily in trace analysis. LC-GC methods have greatly reduced or practically eliminated manual sample-preparation work, allowed to detect traces that could not be analyzed before, and provided results of an accuracy and precision for the whole analysis that often could not be achieved, not even for the final GC step alone.

The range of possibilities in LC sample preseparation or clean-up seems nearly unlimited. Considering that sample preparation constitutes the most time-consuming (thus most costly) part of most chromatographic analyses, this should stimulate further development work in this field. In our laboratory, approximately half of all GC applications now involve on-line $\mathrm{LC}-\mathrm{GC}$, and this proportion will increase as soon as automated methods for the analysis of pesticides, mycotoxins, and veterinary drugs become available.

A fully automated LC-GC system is available from Carlo Erba. A similar instrument is under construction by Chrompack. This is probably just the beginning of more complex instrumentation. On the one hand, such machines could be extended to include on-line derivatization and first sample preparation steps with cartridges that are just used once (for the removal of large amounts of by-products). On the other hand, instruments specially designed for certain purposes (analyzers), such as for trace analysis of organics in water, would certainly find a large market. It would be a pity, if development work in classical chromatography, such as LC and GC, would come to a stop now.

Received: March 18, 1991

[1] K. Grob, 'On-Line Coupled LC-GC', Hüthig, Heidelberg, 1991

[2] H.J.Cortes, C.D. Pfeiffer, B.E. Richter, $H R C C C$ $1985,8,469$.

[3] G.S. Heo, J.K. Suh, HRC 1990, 13,748

[4] K. Grob, Z. Li, J. Chromatogr, 1989, 473, 391

[5] K. Grob, H.P. Neukom, Z. Li, J. Chromatogr. $1989,473,401$

[6] D. Duquet, D. Dewaele, M. Verzele, S McKinley, HRC CC 1988, /1, 824.

[7] H.J. Cortes, C.D. Pfeiffer, G.L. Jewett, B.E. Richter,J.Microcolumn Separations 1989, I,28.

[8] E.C. Goosens, D. de Jong, J.H.M. van den Berg, G.J. de Jong, U.A.Th. Brinkman, J. Chromatogr , in press.

[9] K. Grob, Z. Li, J.Chromatogr. 1989, 473, 423

[10] K. Grob, E. Müller, J. Chronatogr. 1989, 473 , 411.

[II] K. Grob, J. Chromatogr. 1989, 477, 73 .

[12] A.T. Boo, J. Krohn, J. Chromatogr. 1984, 301 335.

113] P. van Zoonen, G.R. van der Hoff, E.A. Hogendoorn, $H R C$ 1990, 13, 483 .

[14] E.C. Goosens, R.G. Bunschoten, V. Engelen, D de Jong, J.H.M. van den Berg, HRC 1990, 13 438.
[15] K. Grob, $H R C 1990,13,540$

[16] J.J. Vreuls, G.J. de Jong, U.A. Th. Brinkman, Chromatographia, in press.

[17] K. Grob, M. Lanfranchi, A. Artho, J.AOAC, in press.

[18] K. Grob, M. Biedermamn, A. Artho, J. Egli, $Z$. Lebensm. Unters. Forsch., in press.

[19] K. Grob, I. Kaelin, A. Artho, $H R C$, in press.

[20] R. Schwarzenbach, J. Chromatogr. 1985, 3.3. 435 .

[21] K. Grob, Z. Li, J. Chromatogr. 1988, 455, 297.

[22] K.Grob, E. Müller, W. Meier, $H R C C C$ 1987, 10 , 416

[23] I.L. Davies, M.W. Raynor, P.T. Williams. G. E. Andrews, K.D. Bartle, Anal. Chem. 1987, 59, 2579.

[24] K. Grob, M. Biedermann, A. Caramaschi. B. Pacciarelli, HRC 1991, 14,33.

[25] S.A. Wise, S.N.Chesler, H.S. Hertz, L.R. Hilpert, W.E. May, Anal. Chem. 1977, $49,2306$.

26] R. Barcarolo, HRC 1990, 13, 465 .

[27] C.E. Werhoven-Goewie. W.M. Boon. A.J.J. Praat, R.W. Frei, U.A.Th. Brinkman.C.J. L.ttle, Chromatographia 1982, 16, 53 .

28] E. Noroozian, F.A. Maris, M.W. F. Nielen, R.W. Frei, G.J. de Jong, U.A.Th. Brinkman, HRC CC 1987, 10.17.

[29] J.J. Vreuls, W.J.G.M. Cuppen, G.J. de Jong, U.A.Th. Brinkman, $H R C$ 1990, 13, 157.

[30] K. Grob, M. Lanfranchi, HRC 1989, 12, 624

[31] W.Specht, M. Tillkes, Fresenius Z.Anal.Chem. $1985,322,443$.

[32] S.J. Chamberlain, Analyst 1990, 115, 1161.

[33] L.G.M.Th. Tuinstra, J.A. van Rhijn, A.H. Roos. W.A. Traag, R.J. van Mazijk, P.J.W. Kolkman, HRC 1990, 13, 797.

[34] M. Minarik, R.J. Oerschkes, G. Wulff, LC.GC Iml. 1990, 3, 51 .

[35] L.G.M.Th. Tuinstra, J.A. van Rhijn, A. Ruiter, Poster at 18 th Int. Symp. on Chromatogr., Amsterdam. Sept. 1990.

[36] T. Takeuchi, T Saito, D. Ishii, J. Chromarogr. 1986, 351, 295.

[37] M. Ghijs, C. Dewacle, P. Sandra, HRC 1990, 13, 651.

[38] M. Ghijs, J. Van Dijck, C. Dewacle, M. Verzele, P. Sandra, Proc. 10th Int. Symp. on Capillary Chromatography, Riva del Garda, 1989, Hüthig, Heidelberg, p. 726.

[39] K.A. Ramsteiner, J. Chromatogr. 1987, 393. 123.

[40] 1.A. Fowlis, $H R C$ 1990, 13. 138

[41] Th.V. Raglione, R.A. Hartwick, .J.Chromatogr. $1988,454,157$.

[42] K. Grob, H.P. Neukom, J. Chromatogr. 1984, $295,49$.

[43] K. Grob, M. Lanfranchi, C. Mariani, J. Clwomatogr. 1989, 471, 397.

[44] K. Grob, M. Lanfranchi, C. Mariani, J.Am. Oil Chem. Soc. 1990, 67, 626. 